UDC: $\left.811.134 .2^{\prime} 373.46\right]: 79$

DOI: https://doi.org/10.18485/beoiber.2018.2.1.5

\author{
Stefan Kovljanin ${ }^{1}$ \\ Doctorando en la Universidad de Belgrado \\ Serbia
}

\title{
EL LENGUAJE Y EL ESTILO DE LA CRÓNICA FUTBOLÍSTICA
}

\section{Resumen}

El presente trabajo tiene como objetivo exponer las características principales, tanto léxicas como estilísticas, morfológicas y sintácticas del lenguaje empleado en las crónicas futbolísticas. La importancia de fútbol como el deporte más seguido en todo el mundo ha hecho que el consumo a diario de los textos relevantes sea enorme, gracias en buena parte al empleo de las nuevas tecnologías en los últimos años.

El trabajo aborda primero el tema de la definición del género de la crónica y sus características formales, y luego se presentan los rasgos lingüísticos más destacados. Dado que se trata de un género especializado, cuya base reside en una terminología específica, se examinan las características principales de los términos desde el punto de vista morfológico y léxico, y después se acude a la presentación de las unidades fraseológicas relacionadas con el argot. Finalmente, se exponen algunos fenómenos sintácticos propios del género, y se hace hincapié en las incorrecciones más frecuentes respecto a la norma actual.

Además, de este modo se intenta presentar una parte de los muchos estudios recientes que abordan diferentes aspectos de la jerga futbolística y que pueden ser muy útiles como base para futuras investigaciones empíricas.

A través de lo expuesto, se nota que el ámbito de la jerga futbolística presenta un campo muy digno de investigación lingüística, sobre todo con la prolífica producción de los textos mediáticos que pueden formar un corpus auténtico, y así permitir un estudio relevante del uso real de la lengua.

Palabras clave: crónica periodística, fútbol, lenguaje específico, estilo, terminología.

\section{LANGUAGE AND STYLE OF FOOTBALL MATCH REPORTS IN SPANISH}

\begin{abstract}
The primary aim of this paper is to present the main lexical, stylistic, morphological and syntactic characteristics of the language used in football match reports of the media in Spanish. Due to the fact that football is the sport with the most followers around the world, in recent years we have witnessed the increase of consumption of relevant texts, especially with the emergence of the specialized media on the Internet.
\end{abstract}

${ }^{1}$ stefan.kovljanin@gmail.com 
The paper first addresses the issue of defining the genre of la crónica futbolistica and its formal aspects, and then we proceed with the presentation of the most prominent linguistic features. Being a specialized genre, it is based on a specific terminology, so we further present the main characteristics of some of the terms from the morphological, lexical and phraseological points of view, based on recent corpus research. Finally, some relevant syntactic phenomena of the genre are mentioned, with emphasis on the most frequent deviations from the standard norm, as it's demonstrated in the literature.

Additionally, the secondary aim of this article is to present the findings of some exhaustive new studies that address different aspects of football slang, as we are convinced that they can be very useful as a basis for future empirical research.

In conclusion, it is evident that the texts on football in general and especially the field of football slang are extremely suitable for any type of linguistic research. The prolific production of media texts helps establishing a broader authentic corpus, and thus facilitates any study of the real language use in this specific domain.

Key words: specific language, style, terminology, match report, football.

\section{Introducción: el periodismo deportivo en el siglo XXI}

La evolución de los medios de comunicación en los comienzos del siglo XXI, especialmente impulsada por el uso de Internet como el medio dominante para un público más joven, ha conllevado varios cambios en la práctica del periodismo. Las nuevas tecnologías han facilitado la penetración de los contenidos hacia los consumidores, de modo que se ha creado un nuevo mercado en el cual cada día luchan varios medios generales y especializados para tener el mayor impacto posible en el público.

Tal como dice Rojas, el periodismo deportivo ha adquirido más protagonismo en los últimos años, así que ahora mismo representa "la tipología informativa de mayor alcance social en una buena parte de los países tanto europeos como americanos" (2014: 178). Las formas comunicativas del deporte han desarrollado además una dimensión que supone aspectos de integración, calidad de vida y trasmisión de valores, $y$, por último, han ampliado su influencia relacionándose con los ámbitos de información, investigación científica, educación, mundo empresarial, etc. (Castañón 2012: 344).

Asimismo, creciendo como ámbito de estudio ha ganado un prestigio del que no disfrutan muchas otras ramas de periodismo. El lenguaje del deporte, sobre todo, es el tema de un número creciente de investigaciones, en buena parte gracias al uso de la red y a la aparición de muchos periódicos especializados, cuyo contenido actual puede ser fácilmente coleccionado e investigado.

Siendo el fútbol el primer deporte que se ha convertido en un espectáculo global y que cuenta con un gran número de personas que lo siguen, no sorprende que el interés en su terminología se remota a los años 70, cuando apareció el primer estudio; hoy en día, como consecuencia, disponemos de muchos estudios exhaustivos, sobre todo en el ámbito de la morfología (véase Nomdedeu 2004). 
Esta contribución se dedica a exponer en un espacio reducido los rasgos más importantes del lenguaje futbolístico empleado en uno de los géneros más habituales del periodismo deportivo - la crónica, partiendo de fuentes teóricas actuales y numerosas investigaciones de corpus de los últimos años.

\section{La crónica periodística y la crónica deportiva}

Los cambios en la práctica del periodismo en la actualidad conllevan, además, cambios en el ámbito de los géneros textuales. Uno de los más populares géneros periodísticos propios de los medios deportivos es el género textual que se continuamente "reinventa por parte de los periodistas" (Rodríguez 2006): la crónica deportiva. Su presencia y transcendencia han conseguido que sea también uno de los géneros más investigados por los lingüistas, sobre todo desde principios del siglo XXI, gracias en mayor medida al aumento de interés en él con la evolución del mercado y al uso de Internet como medio que utiliza cada vez mayor número de personas para mantenerse informados en el día a día.

Dichas investigaciones han tenido como punto de partida diferentes perspectivas o enfoques - discursivo, pragmático, sintáctico, semántico, etc.; sin embargo, aun así, el género carece de unas definiciones y clasificaciones exactas y ampliamente aceptadas debido a su naturaleza híbrida y origen histórico-literario, que le destacan entre los demás géneros periodísticos (Gil 2004: 35-36).

No obstante, la mayoría de los teóricos e investigadores de la crónica (Hernando Cuadrado, Salaverría, Moreno, Grijelmo, Parratt, etc.) la consideran como un género informativo-interpretativo; es decir, su función no es solo explicar los iqué?, iquién?, ¿cómo?, ¿cuándo?, ¿dónde? y ipor qué?, sino que el cronista también debe comentar y/o² juzgar los hechos desde el punto de vista personal y desde la relativa subjetividad, pero evitando opinar sin fundamento. El autor así debe manifestar claramente su presencia activa con la selección de los hechos, la redacción y los comentarios bien justificados que se basan exclusivamente en su conocimiento y experiencia, y sin alteración alguna de la realidad (Gil 2004). Asimismo, según Rodríguez (2006), su tono debe ser más íntimo y de confianza, y, añade Grijelmo (2008: 99), tal que presenta hechos con humildad y así permite a los lectores que formen su propia opinión sobre el asunto. La interpretación en una crónica, según él, se construye utilizando una amplia paleta de verbos, adjetivos y adverbios que matizan lo sucedido: por ejemplo, el uso de los verbos como espetar, resaltar, lamentar, enfatizar frente a asegurar, decir, añadir, agregar claramente ayuda a transmitir una visión más personal y, al mismo tiempo, expresiva y detallada.

${ }^{2}$ La naturaleza de los comentarios y juicios es uno de los principales puntos de discordancia entre los teóricos. Para más detalles sobre todas las controversias del género, véase Rodríguez (2006) o Yanes (2006). 
La implicación del cronista, que suele ser un especialista en la materia, resulta especialmente importante en el subgénero de las crónicas deportivas: numerosos autores destacan que en este tipo el autor tiene aún más libertad para expresar sus juicios, con lo que sus crónicas se parecen más a artículos de opinión. Sin embargo, según Grijelmo (2008: 106-107), en la práctica esto puede llevar a que los cronistas prescindan de narrar lo acontecido, suponiendo que los lectores ya conocen los datos relevantes, y así, bajo el nombre de la crónica, escriben un texto que no pertenece al género y, además, trasmiten opiniones muy discutibles.

Una crónica deportiva también se distingue de las demás por su relativa libertad temática, en el sentido de que, aunque sigue un tema principal, el cronista tiene permiso a abordar diferentes temas laterales, pero relacionados estrechamente con el principal (p.ej. la relación entre los hinchas y la directiva, o la política de los fichajes). Su destreza discursiva aquí debe ser fundamental para que el relato transcurra sin dificultades para el lector.

Para terminar esta parte, cabe mencionar que el proceso de la creación de una crónica deportiva también refleja las peculiaridades del género. Citando a Hernández Alonso, Naranjo (2011: 80-81) destaca algunos rasgos que determinan su desarrollo, además de la extensión y elementos visuales: una crónica se escribe con rapidez, para una pronta publicación, el tono puede variar según la importancia de los protagonistas y el resultado final determina el relato en mayor medida, si hay identificación del periodista con alguno de los equipos.

\subsection{La estructura de la crónica}

En primer lugar, es oportuno constatar que la estructura de la crónica ha sido uno de los aspectos que han recibido muy poca atención hasta ahora, así que en la literatura no hemos encontrado ningún esquema estructural preferente. Autores como Rodríguez (2006) o Yanes (2006) insisten en la libertad estructural, mientras que parece que la organización estructural tripartita (que consta de la presentación, el relato y la conclusión) que propone Moreno (2000) no está documentada en la práctica reciente, sobre todo en el subgénero de las crónicas deportivas.

Debido al espacio reducido que se le otorga normalmente a las crónicas deportivas, frecuentemente la acompañan elementos visuales, como infografías, fichas técnicas, clasificaciones etc. (Rojas 2013). Las restricciones de longitud suponen que el cronista debe poseer una gran habilidad lingüística para cumplir las dos funciones de la tarea: informar de manera concisa y agradable y comentar los hechos aportando juicios valorativos sin pasar el límite entre la interpretación y la opinión. 


\subsection{El estilo de la crónica}

Teniendo en cuenta lo dicho sobre la naturaleza del género, se puede decir que el estilo de una crónica depende exclusivamente del estilo personal del cronista: aunque el elemento noticioso requiere brevedad y claridad de expresión, en la parte interpretativa el autor, guiado por escasos patrones estructurales, tiene toda la libertad de dejar su sello. Hernando (2002: 263) apunta que en los periódicos generales el vocabulario tiende a ser accesible al lector medio: se reducen tecnicismos, extranjerismos, neologismos, vocablos de argot y se prefieren palabras cortas y oraciones simples. Añade que, para lograr dinamismo y evitar monotonía, los autores pueden alternar frases largas y cortas, variar la construcción de las frases y procurar que el discurso se desarrolle sin transiciones repentinas.

Como muestra del buen estilo periodístico, como propone Hernando (2002: 262270) se deben evitar clichés, salvo en casos excepcionales de énfasis, y no se debería imitar el registro administrativo con su tendencia de utilizar circunloquios en vez de palabras únicas, p.ej. dar comienzo en vez de comenzar, poner de manifiesto en vez de manifestar, etc. También, se deben evitar las expresiones que se utilizan como meros añadidos (claro está, como es natural, etc.), o adverbios en -mente cuando sirven de muletillas, y se han de renunciar pleonasmos, siguiendo la tendencia a la economía del lenguaje. Finalmente, se deben evitar los tópicos, p.ej. los abusados empiezan a rodar el balón, los jugadores saltaron al césped, cero a cero, empate sin goles, dar la vuelta al marcador, colocar el balón entre los tres palos, etc. (Naranjo 2011: 86; Díez 2014: 296).

Grijelmo (2008) entre las características del buen estilo nombra la claridad, en el sentido de evitar ambigüedades, la ordenación lógica, elementos de humor, ironía y sorpresa, la selección cuidadosa de adjetivos y metáforas y el ritmo y el sonido, entre otros. En cuanto al mal estilo, Grijelmo apunta la pobreza de la expresión, el lenguaje impreciso y la vulgaridad.

Sin embargo, dado que en la práctica las crónicas futbolísticas permiten un distanciamiento de las normas generales del estilo y de la redacción, el empleo de "las expresiones más rotundas, más grandilocuentes, más exageradas" (Grijelmo 2008: 446) es frecuente, o sea, el sensacionalismo, con el fin de aumentar las tiradas. Aunque todos los investigadores del tema son críticos de esta práctica en la prensa deportiva, muchos concuerdan en que el consumo masivo, desde el punto de vista de la empresa periodística, es el fin que justifica los medios.

Bajo el sensacionalismo, Grijelmo (2008: 543) considera una forma de presentar e interpretar la realidad, sin necesariamente falsificar la información. En la prensa española, apunta, la prensa deportiva sobre todo emplea el sensacionalismo en los titulares y en la presentación de los hechos triviales como fundamentales. No obstante, el empleo de dichas técnicas conlleva inmediatamente varios problemas éticos, especialmente si suponen especulaciones que se presentan como hechos reales. 


\subsection{El lenguaje de la crónica futbolística}

Los textos sobre el fútbol, como señala Gómez, además del léxico característico y de una retórica especial y llamativa, también se caracterizan por unos rasgos gramaticales que difieren del uso normativo de la lengua general. Por lo tanto, insiste en que un estudio profundo del lenguaje del fútbol «deberá tener en cuenta no sólo el léxico con sus campos semánticos, sus sinonimias y antonimias, sus impropiedades léxicas, etc., sino también el plano retórico y el gramatical" (2010: 133).

Las crónicas futbolísticas normalmente son fácilmente entendibles y sencillas para el lector medio, pero se tiene que tener en cuenta que se trata de un periodismo especializado, o sea, que los lectores ya conocen la base teórica y terminológica del tema (Díez 2014). Además, se puede suponer que los lectores de las crónicas en particular son buenos conocedores del asunto, ya que algunos leen la crónica a pesar de haber visto el partido, solo para ver si su opinión coincide con la del cronista.

De esta manera, un lector típico de la crónica sabrá perfectamente qué pasó si en el área chica hubo una plancha al meta, o cómo se sentía un jugador si no le habían salido elásticas ni rabonas, si le habían hecho un caño o si había fallado una cola de vaca, y entiende de maravilla qué tipo de partido se esperaba si se enfrentaron un equipo que aparca el autobús y vive de la pizarra y otro que juega entre líneas, iy además en ese momento padece el virus FIFA!

No obstante, alguna parte del argot del fútbol puede ser entendible también para los no aficionados, dada la penetración que el lenguaje este deporte tiene en la vida cotidiana gracias a su fuerte simbolismo. Así, muchas palabras y expresiones de la jerga futbolística han encontrado camino hacia el vocabulario general y se utilizan a menudo dentro de otros campos técnicos. Así, como documenta García (2002: 34), los ámbitos político y económico son especialmente susceptibles a las expresiones del fútbol: así, p.ej., los políticos se pueden hallar en fuera de juego con sus declaraciones, pueden echar balones fuera cuando están apretados por la prensa, hacer pretemporada antes de las elecciones generales o estar en el banquillo para el nuevo Gobierno. Más allá de las dos esferas mencionadas, cada uno puede recibir una tarjeta amarilla antes de la separación, algunos se casan de penalti y otros se convierten en pichichis de su empresa por su alto rendimiento. Además, los resultados de un partido de fútbol son tan reconocibles que en un espacio muy reducido pueden transmitir mucha información, así que no es de extrañar su uso en los titulares de todo tipo de textos periodísticos.

En las crónicas se hallan frecuentemente expresiones muy emotivas, hipérboles y énfasis con tal de transmitir la pasión con la que el cronista ha vivido el partido. Una de las muestras de la creatividad e invención de los cronistas también es el uso prolífico de las metáforas. En su exhaustivo estudio de las metáforas en el léxico del fútbol en España, Medina $(2007,2009,2015)$ identificó más de 40 campos semánticos diferentes a los que pertenecen las metáforas de su corpus, de los cuales el campo más numeroso fue el 
bélico-militar, dada la representación metafórica estructural en la que "el fútbol es una guerra".

El campo bélico-militar también fue encontrado predominante en el estudio léxico realizado por Arroyo y García (2012) sobre un corpus de textos sobre el Mundial de 2010. Además de ese campo, ellos identificaron léxico de tipos social, lúdico, mágico y jurídico, y mostraron que el sentimiento de victoria o derrota influye fuertemente en la densidad de dichos vocablos dentro de una crónica.

Aparte de la metáfora, no sorprende la existencia de aún más tipos de creaciones lingüísticas, como ahora neologías proverbiales o juegos de palabras (Mapelli 2009). Los juegos de palabras en forma de rupturas de frases hechas o alteraciones de los títulos de libros, películas, canciones, etc. son notables especialmente en los titulares (p.ej. A Dios rogando... y el Mundial ganando; Crónica de una victoria anunciada; Operación Cardiff, etc.), ya que las creatividades como estas suelen cautivar la atención instantáneamente (véase Mapelli 2004).

\subsection{La terminología del fútbol}

Naranjo (2011: 86-88) insiste en que el lenguaje deportivo abunda en tecnicismos que, sin embargo, no dificultan mucho su comprensión, dado que se asimilan con facilidad y llegan a formar parte del vocabulario propio de los lectores. Dentro del lenguaje futbolístico, destacan términos que designan a los participantes, las partes del terreno, las fases del juego o algunas acciones y situaciones específicas, y a menudo existen muchos sinónimos para un mismo término. Así, al portero se le llama también guardameta, arquero o cancerbero; en la defensa o zaga hay centrales, laterales, líberos, carrileros, en el centro del campo (mediocampo) hay interiores, extremos, centrocampistas de contención, pivotes, etc., y en la parte más ofensiva además de atacantes figuran arietes, puntas, mediapuntas, enganches, trecuartistas, nueves falsos, etc.

En uno de los estudios más extensivos de la terminología del fútbol, Nomdedeu (2004) ha investigado los diferentes procedimientos de creación de los términos futbolísticos y ha identificado la neología, la variación denominativa, procedimientos morfológicos, procedimientos semánticos, fenómenos sintácticos y otros procedimientos. En los siguientes párrafos intentaremos presentar sus resultados en lo más breve.

Primero, él destaca la productividad de lo que denomina variación (casos de sinonimia) y lo apoya con ejemplos de su corpus de tipo penal - de penalty o penalti, para la variación morfológica; bajo la variación léxica agrupa ejemplos de variación diatópica, tanto en el español peninsular (tipo árbitro - colegiado), como entre el peninsular y el de América (tipo campo - cancha); finalmente, bajo la variación por préstamo de otras lenguas agrupa ejemplos como blaugrana - azulgrana.

En el apartado sobre los procedimientos morfológicos, Nomdedeu (2004) analiza el comportamiento de varios prefijos y sufijos productivos en este campo. Así hay tres 
prefijos de negación empleados a menudo (anti-, contra- $y$ des-), un prefijo temporal (pre-), uno de cantidad y tamaño (semi-), dos de intensificación (re- y super-) y un prefijoide (auto-).

Cuando se trata de la sufijación en la creación de los sustantivos, Nomdedeu (2004) identificó algunos casos de sufijación apreciativa (p.ej. mundialito, liguilla, paradón, zapatazo, etc.) y muchos más casos de la sufijación no apreciativa, que se ha manifestado como el tipo más productivo en su corpus. De esta manera encontramos ejemplos de sufijación denominal (p.ej. pañolada, plantel, linier, espinillera, esférico, colista, etc.), deadjetival (p.ej. triplete, titularidad), deverbal (cantada, pegada, marcaje, triangulación, lanzamiento, etc.) y deadverbial (p.ej. delantero).

Como un fenómeno bastante relevante su estudio identifica la derivación regresiva (derivación posverbal o sufijación cero), que se manifiesta cuando la palabra derivada consta de menos letras que la primitiva (casos como despeje, enganche, entreno, rechace, regate, etc.).

La creación de adjetivos y verbos no es tan prolífica como en el caso de la nominalización. En cuanto a la adjetivación, Nomdedeu (2004) ha identificado 6 sufijos productivos (p.ej. canterano, medular, sevillista, futbolístico, etc.), y, mientras tanto, los casos de verbalización comprenden 4 sufijos productivos (presentes en: retratar, gambetear, materializar, profundizar, etc.).

La formación parasintética también está representada en su corpus, aunque en menor medida que la derivación pura (p.ej. alinear, ensanchar).

La creación de vocablos mediante la composición también está muy presente dentro del corpus investigado por Nomdedeu (2004). Así, hay un número importante de casos de compuestos ortográficos que pertenecen a cinco diferentes tipos: nominal (mediapunta), verbal + nominal (guardameta), adjetivos compuestos (albiceleste), compuestos por acronimia (Eurocopa) y compuestos reduplicativos (extremo-extremo).

Otra aportación reciente de importancia al tema de la creación del léxico de fútbol es el trabajo de Gómez (2010).

Cuando se trata de la prefijación, él apunta el uso de los prefijos semi- y vice-con el remoto significado de aproximación o de algo inmediatamente inferior (vicegol, semicórner). Además, entre los más productivos destaca los prefijos auto- (autopase), co(colíder), pre- y post- (prepartido, postpartido), anti- (antifútbol), contra- (contragolpe) y super- (superequipo).

En cuanto a la sufijación, Gómez (2010) nombra varios sufijos productivos, entre los cuales destaca la productividad del sufijo deverbal - e en los neologismos (p.ej. saque, pase, rechace, cierre, etc.). Con el sufijo -ada aparecen palabras con significados de acción (parada, estirada), de golpe (pegada), abundancia (goleada) o cualidad (cantada). Los dos valores del sufijo -azo también aparecen en la jerga futbolística: de golpe (pelotazo, cabezazo) y como aumentativo en el sentido cualitativo (golazo, partidazo). La intensificación se consigue mediante el sufijo -ón, como en las palabras entradón o jugadón. Por último, la productividad del sufijo -ero/a es igualmente visible en el ámbito 
de fútbol que en la lengua general: lo confirman palabras que designan posiciones (portero, zaguero) u otras donde el sufijo aporta rasgos de calidad (p.ej. leñero, tarjetero). Igual que el sufijo -ero/a en la derivación nominal funciona el sufijo -ista, pero también sirve para la creación de nuevos adjetivos que designan jugadores o aficionados (madridista, barcelonista, sevillista, etc.).

Sin un análisis muy amplio, Gómez (2010: 138) también nombra algunos casos de composición: p.ej. guardameta, medialuna, mediapunta, etc.

Cuando se trata de las unidades léxicas compuestas del ámbito futbolístico, tampoco faltan investigaciones recientes. Primero, nos remitimos otra vez al trabajo de Nomdedeu (2004), quien ha identificado 426 unidades pluriverbales de varios grupos de las que nombraremos solo los más grandes: binominales (fútbol base, hombre gol), con preposición (línea de cobertura, uno contra uno), del tipo $\mathrm{N}+$ Adj (balón mordido, tiempo reglamentario), locuciones adverbiales (a puerta vacía, por la mínima), locuciones verbales (ganar la espalda, jugar al pelotazo), etc.

En otro relevante estudio, Palau (2007) concluye que las colocaciones del tipo $\mathrm{N} /$ Adj $+\mathrm{N}$ a menudo se utilizan para caracterizar positiva $\mathrm{O}$ negativamente a los protagonistas o el juego. En su corpus de investigación se encuentran, p.ej. expresiones clara ocasión, extrema facilidad, aplastante dominio, garrafal error, nefasta racha, etc. Las colocaciones hechas por un adjetivo o participio adjetivizado y un adverbio de modo o un verbo y adverbio, en su opinión sirven para intensificar y así apelar al lector. Así, en una crónica se pueden hallar expresiones como infinitamente superior, totalmente justo, justamente expulsado, fallar estrepitosamente, resolver magistralmente, cambiar drásticamente, etc.

En cuanto a las locuciones, en el corpus seleccionado por Palau (2007: 204-206) aparecen locuciones verbales que designan acciones específicas, como hacer una internada, despejar un balón, jugar por las bandas, etc. igual que algunas expresiones coloquiales de este tipo: echar por tierra, apretar más los dientes, sudar sangre, jugar con fuego, etc. Por otra parte, las locuciones verbales sirven al cronista para valorar: p.ej. se inventó diagonales a la perfección, defiende de cine, funciona de lo lindo, de milagro a milagro sobrevive, perdonó en demasía, etc.

En su estudio sobre las locuciones del lenguaje del fútbol, Mapelli (2004) encuentra que existe cierta variabilidad en algunas de las locuciones observadas y que las locuciones verbales son las más numerosas, porque designan acciones del juego (p.ej. saltar al terreno, achicar espacios, adelantar líneas, despejar el balón, etc.). Las adjetivales y adverbiales también son frecuentes (a las nubes, a la contra, al hombre, en corto, etc.), mientras que el grupo de las nominales no es tan numeroso (pase de la muerte, gol fantasma, palo corto, sequía goleadora, etc.).

Como ya hemos señalado, es frecuente el uso de la metáfora y la metonimia en los textos sobre el fútbol. Por lo tanto, es de esperar que los procedimientos semánticos en la formación de la terminología futbolística también sean productivos. Cuando se trata de la 
metáfora, Nomdedeu (2004) ha encontrado 34 campos semánticos con los que se relacionan nuevas palabras, las que él considera términos del dicho ámbito profesional. Entre los campos predominan el mundo bélico, la muerte, la construcción, el derecho, los medios de transporte y el cuerpo humano (p.ej. cañonear, fusilar, disparar, punto fatídico, fabricar un gol, hacer un túnel, sentenciar el partido, tener olfato de gol, etc.).

La metonimia también destaca por el número de ejemplos en el corpus investigado por Nomdedeu (2004). Entre esas palabras así generadas predominan las designaciones de los jugadores por su demarcación (arquero, central, nueve, zaguero, etc.), pero también hay otras, por ejemplo a partir de la relación hecho-lugar donde se produjo, u objeto-cualidad (p.ej. lateral, cuero, esférico, etc.).

A partir de lo expuesto, se nota que la terminología futbolística es especialmente rica en sinónimos, pero también se tiene que tener en cuenta que existen muchos falsos amigos, dada la llamativa variación dialectal. Así, p.ej. golero en Ecuador o Paraguay significa 'guardameta', y en la mayoría del resto de Hispanoamérica significa 'goleador'. También, a partir de la lista de términos que nos presenta Castañón (2016), se nota que existen diferentes patrones de creación de palabras y que la productividad de algunos sufijos es diferente. Por ejemplo, en español peninsular es frecuente el uso del término once con significado de 'alineación'; sin embargo, en algunas partes de Hispanoamérica, además de este término se utilizan términos derivados: oncena y onceno (2016: 15).

\subsection{Una mención a la sintaxis}

Cuando se trata de las peculiaridades sintácticas de la jerga futbolística, inmediatamente saltan a la vista la elipsis, el dominio del sintagma nominal y el abuso de los atributos. Además, Gómez (2010) nombra otros fenómenos que, según él, merecen un análisis profundo.

Primero, menciona la abundancia de las estructuras apositivas (p.ej. fútbol control, gol fantasma, jugador clave, etc.) y de las sustantivaciones de adjetivos y cardinales (el mano a mano, un 4-4-2, etc.). Igualmente frecuentes son las locuciones que construyen unidades sintácticas y semánticas fijas (pase de la muerte, saque de puerta, despeje de puños, a balón parado, en vertical, de bella factura, de espuela, etc.) y, también, en los textos deportivos se pueden hallar ejemplos de adverbialización de adjetivos (rematar fácil, jugar limpio, entrar fuerte, etc.).

Luego, Gómez insiste en algunas discrepancias con la norma: por ejemplo, en ciertos casos de metonimia donde no hay concordancia en el sintagma (p.ej. extremo izquierda, interior derecha). El rasgo gramatical que destaca (2010: 141) como el preocupante por su intrusión en la lengua estándar es la omisión de artículos exigidos sintácticamente, como en los siguientes casos: sacar bajo palos, estar en túnel de vestuarios, jugar en Liga, etc.

Además, apunta varias anomalías en cuanto al régimen de ciertos verbos, y aporta ejemplos actuales de los textos periodísticos con su uso no estándar: p.ej. entrar ('el 
defensa entró con dureza al delantero'), cabecear ('cabeceó el balón), bailar ('un equipo baila al otro), peinar ('el ariete peinó el balón), pitar ("pitar un partido'), luchar/pelear ('peleó el balón'), circular ('los jugadores circulaban el balón'), encajar ('Rivaldo le encajó tres goles al portero'), etc. (2010: 141-146).

También, propias del argot futbolístico son las estructuras impersonales de tipo 'es penalti o 'ha sido falta', restringidas en el estándar, y la omisión del CD porque se sobreentiende (p.ej. con los verbos marcar, definir, remontar, controlar). Finalmente, según Gómez, es notable el uso de algunas preposiciones que no corresponde con la norma: aquí destacan el uso extensivo de la preposición sobre en vez de de, a o hacia, y de la preposición de con supresión del artículo en lugar de desde, por lo que estos casos se consideran como locuciones o semilocuciones verbales (p.ej. sacar de fondo; Gómez 2010: 148).

Hernando (2002: 271-272) también insiste en que en los textos deportivos existen muchas más incorrecciones, tanto en los aspectos temporal y aspectual, como en el uso de diferentes categorías de palabras, algunas veces de forma sistemática. Así, entre otros, se pueden encontrar casos del uso de pretérito perfecto por el indefinido ("...el fin de semana pasado se han producido...'), o del uso del imperfecto de subjuntivo por el pluscuamperfecto de indicativo en las relativas ('La sesión, que comenzara a las cuatro de la tarde, se prolongó...'), o del anglicismo estar siendo + participio ('La oferta está siendo estudiada...'). Finalmente, apunta Hernando Cuadrado, se registran casos de dequeísmo y de las ambigüedades producidas por la separación de los complementos de término (p.ej. Le encargaron de que, si notaba algo extraño, avisara a la policía o El taxi se encontraba en las afueras de la ciudad, cerca da un edificio destinado a almacén con las puertas abiertas).

\section{Conclusiones}

A partir de lo presentado resulta obvio que la crónica futbolística, como uno de los géneros deportivos predominantes en la actualidad, posee suficiente riqueza de rasgos lingüísticos para que se mantenga en el foco de la investigación desde el principio del siglo. Además, con el aumento del consumo de contenidos de todo tipo relacionados con la práctica social del fútbol y con el crecimiento del número de los medios generales y especializados que tratan el tema de fútbol en detalle, podemos anticipar que seguirá siendo así, especialmente por la facilidad de componer un corpus con ejemplos de uso de lengua reales.

El lenguaje futbolístico muestra particularidades en varios planos, sobre todo en cuanto a los procedimientos de la creación de su terminología y en cuanto al uso de la metáfora como el máximo mecanismo expresivo. Teniendo en cuenta el valor simbólico de este lenguaje, se puede esperar que continúe su expansión hacia el vocabulario 
general, y los promotores de esta expansión indudablemente serán los medios de comunicación, por su relación recíproca con el deporte.

Por lo tanto, es necesario que los periodistas respeten normas éticas de su profesión y que sean aptos para la expresión y redacción de textos, para que el periodismo deportivo en el futuro dé la vuelta al marcador en su duelo particular con el sensacionalismo y que la riqueza de la expresión remate a placer en cada oportunidad de definir para aumentar distancias entre el buen y mal estilo.

\section{BIBLIOGRAFÍA}

Arroyo Almaraz, Isidoro y Francisco García García. “El léxico deportivo de las crónicas periodísticas del Mundial de fútbol 2010 ganado por España en El Poema de Mío Cid». Historia y Comunicación Social, 17 (2012): 317-341. Web. 25 Sep. 2017.

Castañón Rodríguez, Jesús. «El lenguaje periodístico del deporte en el idioma español del siglo XXI». Historia y Comunicación Social, 17 (2012): 343-358. Web. 23 Sep. 2017.

- Guía del Lenguaje Deportivo. Idioma y deporte [en línea]. 1 de enero de 2016, número 179. Web. 23 Sep. 2017.

Díez Yagüe, Roberto. “La crónica como género interpretativo de Enric González: análisis de las historias del calcion. Tesis doctoral. Universidad Complutense de Madrid, 2014. Web. 20 Sep. 2017.

García Molina, Emilio Tomás. Deporte y lenguaje. Madrid: Consejo superior de deportes, 2002. Web. 15 Sep. 2017.

Gil González, Juan Carlos. "La crónica periodística. Evolución, desarrollo y nueva perspectiva: viaje desde la historia al periodismo interpretativo". Clobal Media Journal, 1 (2004): 26-39. Web. 23 Sep. 2017.

Grijelmo, Álex. El estilo del periodista. Madrid: Taurus, 2008. Impreso.

Gómez Torrego, Leonardo. "Aspectos gramaticales del lenguaje del fútbol”. Las lenguas de especialidad y su enseñanza, Ed. Lourdes Díaz, 11 (2010): 132-149. Web. 11 Sep. 2017.

Hernando Cuadrado, Luis Alberto. "Sobre la configuración lingüística del mensaje periodístico". Estudios sobre el Mensaje Periodístico, 8 (2002): 267-274. Web. 22 Sep. 2017.

Mapelli, Giovanna. "Locuciones del lenguaje del fútbol". Atti del XXI Congresso dell'AISPI, Letteratura della memoria. La memoria delle lingue: la didattica e lo studio delle lingue della penisola iberica in Italia. Messina: Lippolis, 2004: 171-181. Web. 10 Sep. 2017.

-. "La prima pagina del Marca: specchio della creatività del linguaggio del calcio". Beatriz Hernán-Gómez Prieto (ed.), Il linguaggio dello sport. La comunicazione e la scuola, Milano: LED, 2009. Web. 10 Sep. 2017. 
Medina Montero, José Francisco. "La metáfora en el léxico futbolístico: el caso de los participantes en español, y sus posibles equivalentes en italiano". Luis Luque Toro (ed.), Léxico Español Actual, Actas del l Congreso Internacional de Léxico Español Actual, Venecia: Libreria Editrice Cafoscarina, 2007: 197-239. Web. 15 Sep. 2017.

- "La metáfora en el léxico futbolístico: el caso de la actividad deportiva en español, y algunas propuestas de traducción al italiano". Luis Luque Toro (ed.). Léxico Español Actual II. Venecia: Università Ca'Foscari, 2009: 155-202. 15 Sep. 2017.

- "La metáfora en el lenguaje futbolístico: el caso del tiempo, el lugar y los útiles deportivos en español, y propuestas de traducción al italiano". Rivista internazionale di tecnica della traduzione, 17 (2015): 137-155. Web. 15 Sep. 2017.

Moreno Espinosa, Pastora. "Los géneros periodísticos informáticos en la actualidad internacional». Ámbitos, 5 (2000). Web. 21 Sep. 2017.

Naranjo de Arcos, Alicia. "Tratamiento de la información deportiva en la prensa: la crónica como género prevalente. El caso de los encuentros de fútbol entre Real Madrid y F.C. Barcelona". Tesis doctoral. Universidad de Málaga, 2011. Web. 12 Sep. 2017.

Nomdedeu Rull, Antoni. "Terminología del fútbol y diccionarios: elaboración de un diccionario de especialidad para el gran público". Tesis doctoral. Universidad Autónoma de Barcelona, 2004. Web. 12 Sep. 2017.

Palau Sampio, Dolors. “Fraseolgia i modalització en la crónica futbolística”. Quaderns de Filologia. Estudis Lingüistics, 12 (2007): 193-210. Web. 19 Sep. 2017.

Rodríguez Betancourt, Miriam. "La crónica periodística: un género tan polémico como imprescindible». Revista Universidad de La Habana, 1 (2006). Web. 13 Sep. 2017.

Rojas Torrijos, José Luis. "Periodismo deportivo. Hacia la innovación y el emprendimiento en la red". Presente y futuro en el periodismo especializado. Madrid: Fragua, 2013: 197-247. Web. 20 Sep. 2017.

- "Periodismo deportivo. Nuevas tendencias y perspectivas de futuro". Correspondencias \& Análisis, 4 (2014): 179-192. Web. 20 Sep. 2017.

Yanes Mesa, Rafael. "La crónica, un género del periodismo literario equidistante entre la información y la interpretación". Espéculo. Revista de estudios literarios, 32 (2006). Web. 23 Sep. 2017.

Fecha de recepción: 15 de enero de 2018. Fecha de aceptación: 14 de abril de 2018. 